\title{
The outcomes in STEMI patients with high thrombus burden treated by deferred versus immediate stent implantation in primary percutaneous coronary intervention: a prospective cohort study
}

\author{
Demou Luo ${ }^{1}$, Xiangming Hu ${ }^{1}$, Shuo Sun ${ }^{1}$, Chenyang Wang ${ }^{1}$, Xing Yang ${ }^{2}$, Jingguang Ye ${ }^{2}$, Xiaosheng Guo ${ }^{2}$, \\ Shenghui $\mathrm{Xu}^{2}$, Boyu Sun ${ }^{2}$, Haojian Dong ${ }^{1}$, Yingling Zhou ${ }^{1}$ \\ ${ }^{1}$ Guangdong Cardiovascular Institute, Guangdong Provincial People's Hospital, Guangdong Academy of Medical Sciences, Guangzhou, China; \\ ${ }^{2}$ Department of Interventional Treatment, Guangdong Provincial People's Hospital Zhuhai Hospital, Zhuhai, China \\ Contributions: (I) Conception and design: Y Zhou, H Dong; (II) Administrative support: D Luo, S Sun, X Hu; (III) Provision of study materials or \\ patients: X Yang, B Sun, C Wang; (IV) Collection and assembly of data: J Ye, X Guo, S Xu; (V) Data analysis and interpretation: Y Zhou, H Dong, D \\ Luo; (VI) Manuscript writing: All authors; (VII) Final approval of manuscript: All authors. \\ Correspondence to: Yingling Zhou. Guangdong Cardiovascular Institute, Guangdong Provincial People’s Hospital, Guangdong Academy of Medical \\ Sciences, 96 Dongchuan Road, Guangzhou 510080, China. Email: zylgdh@163.com; Haojian Dong. Guangdong Cardiovascular Institute, \\ Guangdong Provincial People’s Hospital, Guangdong Academy of Medical Sciences, 96 Dongchuan Road, Guangzhou 510080, China. \\ Email: donghaojian@sina.com.
}

Background: No-/slow-reflow indicates worse outcomes in ST-elevation myocardial infarction (STEMI)
patients with high thrombus burden. We examined whether deferred stenting (DS) strategy reduces no-/
slow-reflow or major adverse cardiovascular events (MACEs) in primary percutaneous coronary intervention
(pPCI) for patients with acute STEMI and high thrombus burden.
Methods: We performed an open-label, multi-center, prospective cohort study among eligible patients
with acute STEMI and high thrombus burden who further received pPCI. All participants received PCI with
DS (second procedure performed within $48-72$ h) or immediate-stenting (IS) strategy. The primary outcome
was the incidence of no-/slow-reflow. We evaluated MACEs and bleeding events during hospitalization and
at 30 - and 90-day follow-ups. Results: We recruited 245 patients to this study, including 51 with DS and 194 with IS. Baseline clinical characters were comparable between the 2 strategies. Incidence of no-/slow-reflow defined by thrombolysis in myocardial infarction (TIMI) flow grade was not significantly different between the 2 strategies [DS: 5 (9.8\%), IS: 33 (17.0\%), P=0.21]. No-/slow-reflow by TIMI myocardial perfusion grade (TMPG) was less prevalent in DS [20 (39.2\%) vs. 107 (55.2\%), $\mathrm{P}=0.04]$. No significant differences were found in recurrence of myocardial infarction $(\mathrm{P}=0.56)$, cardiac death $(\mathrm{P}=0.37)$, all-cause mortality $(\mathrm{P}=0.37)$, heart failure-induced readmission $(\mathrm{P}=0.35)$, or bleeding $(\mathrm{P}=0.61)$ between the 2 strategies in-hospital, and at 30- and 90-day follow-up.

Conclusions: In STEMI patients with high thrombus burden who underwent pPCI, DS strategy reduced no-/slow-reflow of microcirculation. However, DS strategy did not reduce incidence of MACEs or bleeding.

Keywords: ST-elevation myocardial infarction (STEMI); high thrombus burden; deferred stenting; microcirculation; major adverse cardiac events (MACEs)

Submitted Feb 05, 2021. Accepted for publication Apr 01, 2021.

doi: 10.21037/atm-21-1130

View this article at: http://dx.doi.org/10.21037/atm-21-1130 


\section{Introduction}

Primary percutaneous coronary intervention (pPCI) has been validated with abundant evidence and is currently widely used in acute ST-elevation myocardial infarction (STEMI) $(1,2)$. Despite timely reopening of infarct-related artery (IRA), $10-29 \%$ of patients with STEMI could still suffer from complications of no-/slow-reflow after pPCI $(3,4)$. There are several risk factors for the occurrence of no-/slow-reflow, including very high thrombus burden, embolization-related microvascular disturbance, endothelium dysfunction, and myocardial edema. Previous studies have shown that despite the patency of IRA and reperfusion to a thrombolysis in myocardial infarction (TIMI) flow grade of 2-3 after percutaneous transluminal coronary angioplasty (PTCA) with or without thrombus aspiration, the occurrence rate of no-/slow-reflow might increase in STEMI patients treated with an immediate stenting (IS) strategy when compared with a delayed stenting (DS) strategy, resulting in a potentially worse prognosis in myocardial perfusion, heart function, or major adverse cardiac events (MACEs) (5-7). Similar results have been shown in several trials (8), indicating that the instability of thrombus as well as vulnerable plaque would be exacerbated due to IS implantation, with mechanical compression possibly causing microvascular embolism. An effective solution may be a strategy of DS in selected patients following a certain period of time with sufficient antithrombotic therapy when reperfusion is achieved (6-8). However, other trials have found that, compared with an IS strategy in STEMI patients, a planned or selected DS implantation may not improve the outcomes of MACEs (9-11).

Considering the pathophysiological mechanisms of STEMI with high thrombus burden as well as conflicting results from various clinical trials, it remains unclear whether the DS strategy would improve the outcomes in STEMI patients with high thrombus burden. Herein, we hypothesized that the DS and IS strategies would influence the outcomes in STEMI patients with high thrombus burden, and investigated whether the DS strategy would be a better choice than IS for such patients. We present the following article in accordance with the STROBE reporting checklist (available at http://dx.doi.org/10.21037/atm-21-1130).

\section{Methods}

\section{Study design and setting}

This study was designed as a multi-center, open-label, and prospective cohort study. It was conducted at 3 cardiovascular centers in South China (Guangdong Provincial People's Hospital, Guangzhou City; Guangdong Provincial People's Hospital Zhuhai Hospital, Zhuhai City; and Jiexi County People's Hospital, Jiexi City) between January 2018 and January 2020.

Patients with a confirmed diagnosis of acute STEMI and indications for pPCI were invited to participate in our study. Antiplatelet agents, statins, and other evidencebased treatments were routinely used after the obtainment of informed consent. According to the guidelines and clinical situation, pPCI was regularly performed, while the strategies of stent implantation (DS/IS) were determined at the discretion of operators (blinded to the trial) based on the clinical condition as well as contemporary guidelines. Follow-ups were conducted at 30 and 90 days after pPCI. There were no additional interventions from investigators during the entire study. The research was approved by the Ethics Committee of Guangdong Provincial People's Hospital [GDREC2018346H(R2)]. All procedures performed in this study involving human participants were in accordance with the Declaration of Helsinki (as revised in 2013).

\section{Participants}

Patients included in the study had to meet all of the following criteria: (I) age $\geq 18$ years and diagnosis of acute STEMI; (II) planning to receive recommended pPCI; (III) imaging manifestations of high thrombus burden during operation through angiography. High thrombus burden was defined as Thrombus Score (TS) $>2$, which was defined previously by the TIMI study group (12): $0=$ no thrombus; $1=$ haziness; $2=$ definite thrombus $<1 / 2$ vessel diameter; $3=$ definite thrombus $1 / 2$ to 2 vessel diameters; and $4=$ definite thrombus $>2$ vessel diameters; (IV) a TIMI flow grade of 2 to 3 was achieved after primary intervention before stent implantation; and $(\mathrm{V})$ informed consent was provided.

Patients with the following criteria were excluded from the study: (I) cardiogenic shock with or without cardiac arrest; (II) IRA caused by in-stent occlusion/restenosis or saphenous vein bypass graft (SVGs) occlusion/stenosis; (III) IRA dissection; (IV) history of contrast allergy; (V) life expectancy $<12$ months; (VI) Patients with recent active bleeding; (VII) left main artery as IRA.

Investigators also set several eliminating criteria that were unobtainable before admission to exclude confounding bias: (I) left ventricular ejection fraction (LVEF) $<30 \%$; (II) 
hemoglobin (HGB) in plasma $<70 \mathrm{~g} / \mathrm{L}$; (III) the platelet (PLT) count in plasma $<50 \times 10^{9} / \mathrm{L}$.

Participants had rights to withdraw from the study at any time for personal reasons during the research and subsequent follow-up period, without cessation of the standardized treatment. Meanwhile, participants with poor compliance were excluded (by investigators to ensure the accuracy of the results).

\section{Diagnosis of STEMI}

Clinical diagnosis of STEMI was confirmed when 2 out of the 3 following standard criteria were met: (I) typical signs of ST-elevation in 2 contiguous leads on electrocardiogram (ECG); (II) typical signs of elevated cardiac biomarkers including troponin, creatine kinase (CK), and creatine kinase-myocardial band (CK-MB), which were raising proportionally; (III) typical symptoms.

\section{Assessment of coronary angiography (CAG) and intervention}

CAG was performed via the radial or femoral access by experienced operators from the Department of Cardiology in line with the procedural instructions and guidelines. Interventional instruments including angiographic catheters, guiding catheters, guidewires, balloons, and stents were chosen by operators for each participant specifically. To rescue the viable myocardium in participants with STEMI, the pPCI was performed within $12 \mathrm{~h}$ from the onset of symptoms, or after $12 \mathrm{~h}$ in severe conditions such as refractory heart failure, hemodynamic instability, electrophysiological instability, or persistent ischemia. After primary intervention, TIMI flow grade 2 to 3 was achieved before stenting through IS or DS strategy. During the procedure, DS or IS strategy was chosen by the operator depending on the guidelines and their experience. In patients with DS, a second angiography was performed within 48-72 h after the initial coronary reperfusion.

Significant stenosis was defined as at least $50 \%$ filling defect of the reference lumen diameter, while occlusion was defined as a total filling loss with blockage of IRA. Thrombus score was measured. Meanwhile, TIMI myocardial perfusion grade (TMPG) was classified into 4 grades: $0=$ failure of dye to enter the microvasculature; $1=$ dye slowly enters but fails to exit the microvasculature (ground-glass appearance or opacification of the myocardium in the distribution of the culprit lesion that fails to clear from the microvasculature, and dye staining is present on the next injection $<30 \mathrm{~s}$ ); $2=$ delayed entry and exit of dye from the microvasculature (dye strongly persists after 3 cardiac cycles of the washout phase and either does not or only minimally diminishes in intensity during washout); 3 = normal entry and exit of dye from the microvasculature (dye is gone or is mildly/moderately persistent after $\mathrm{t} 3$ cardiac cycles of the washout phase and noticeably diminishes in intensity during the washout phase).

\section{Baseline information and assessment of follow-up}

Medical history information including the onset of symptoms and the quality, location, and severity of chest pain was collected after the provision of informed consent. Demographic statistical data including age, gender, and history of smoking were gathered. Presence of comorbidities such as previous myocardial infarction (MI), heart failure with or without classification by New York Heart Association (NYHA), history of hypertension, diabetes mellitus, chronic kidney disease (CKD), stroke, and bleeding were noted from the medical records. We provided standard medications, including antiplatelet drugs, oral anticoagulants, thrombolytics, angiotensin-converting enzyme inhibitors or angiotensin receptor blockers (ACEIs/ ARBs), calcium channel blockers (CCBs), $\beta$-blockers, proton pump inhibitors (PPIs), nitrates, diuretics, statins, as well as antidiabetic drugs.

\section{Physical examination and supplementary test}

Data on vital signs and routine physical examination such as height, weight, body mass index (BMI), blood pressure (BP), heart rate (HR), respiratory rate (RR), and crackles in lung auscultation were collected at baseline, which could be utilized in evaluating the state of abnormality. Moreover, 18-lead ECG, cardiac echocardiography, routine cardiac biomarkers such as troponin $\mathrm{I} / \mathrm{T}$ (either sensitive or routine one), $\mathrm{CK}, \mathrm{CK}-\mathrm{MB}$, brain natriuretic peptide (BNP)/NTpro-BNP, blood routine culture, and parameters of renal and liver functions were taken to evaluate the state of the disease. The standardized medical therapy in terms of guidelines and routine strategies was also recorded.

\section{Study endpoints}

The primary endpoint of this study was the incidence of no-/ slow-reflow events classified by TIMI flow grade or TMPG, after implantation of stents (participants with IS or DS) or 
PTCA (participants with DS) in IRA. Within this study, the no-reflow phenomenon was defined as obviously decreased or lost blood flow corresponding to degrees 0 or 1 in TIMI flow grade classification, while slow-reflow was considered if there was degree 2 in TIMI flow grade classification. A TMPG $\leq 2$ was also considered as no-/slow-reflow.

This study also focused on the following secondary endpoints: (I) incidence of MACEs (including reinfarction, cardiac death, all-cause mortality, heart failure-induced readmission and revascularization) in-hospital and at 30- and 90-days' follow-up after pPCI; (II) quantitative evaluation of heart function through echocardiography conducted in-hospital after pPCI; (III) Total expenses during hospitalization; (IV) Bleeding events according to Bleeding Academic Research Consortium (BARC) classification (13).

\section{Sample size}

According to previous studies (1-5), it was estimated that with an inclusion period of 2 years and a minimum followup of 90 days, the incidence of no-/slow-reflow could be up to $29 \%$ in IS strategy and $6 \%$ in DS, respectively. Considering a $20 \%$ loss of follow up, a total of 106 participants were required to achieve $80 \%$ power of the study, with a 2-sided type I error of 0.05 .

\section{Statistical analysis}

All analyses were performed using SPSS software (version 23.0, IBM Corp., Armonk, NY, USA). Continuous variables were expressed as mean \pm standard deviation (for normally distributed) or median/quartiles (for those with skewed distribution), and categorical variables were shown as frequencies with percentages. To determine the significance of differences between the groups, $t$-test/one-way analysis of variance (ANOVA) (for normal distribution), MannWhitney $\mathrm{U}$ test (for skewed distribution), and chi-squared test/Fisher's exact test (for categorical variables) were used. Comparisons with $\mathrm{P}<0.05$ (2-sided) were considered statistically significant.

\section{Results}

A total of 245 consecutive patients were enrolled between January 2018 and January 2020 at 3 hospitals in southern China. Data on baseline characteristics were well-matched between the groups, with the exception of percentage of stenosis of ischemic target lesion (Table 1). The planned follow-up of participants was 30 and 90 days. All participants received standardized medical treatment based on the clinical guidelines and physician's judgment. Among the participants, 194 were treated with the IS strategy, while 51 were subjected to the DS strategy $(78.2 \%$ vs. $20.6 \%$, respectively).

Frequency of stent implantation was significantly lower in DS strategy participants (30/51, 58.9\%) compared with those with IS strategy $(\mathrm{P}<0.001)$, while $8.6 \%$ of participants were not stented. A single (0.5\%) IS strategy participant underwent revascularization within $12 \mathrm{~h}$ after the first procedure of IRA due to ischemia of non-IRA. A single DS strategy $(2.0 \%)$ participant received urgent PCI in advance because of repeated ischemia of IRA. A total of 21 DS strategy participants $(41.2 \%$; $8.6 \%$ of the entire sample) did not receive stent implantation owing to satisfactory blood flow (TIMI flow grade 3 ) and mild to moderate percentage of stenosis $(<75 \%)$ according to the planned secondary angiography.

\section{Primary endpoint}

As shown in Table 2, the primary endpoint, which was no-/slow-reflow (TIMI flow grade 0-2) occurred in $33(17.0 \%)$ IS strategy participants and 5 (10.2\%) DS strategy participants (Tables 2,3, Figures 1,2). No significant difference was found between participants of the 2 strategies $(\mathrm{P}=0.21)$. When using TMPG as an alternative primary endpoint, the no-/slow-reflow (TMPG flow grade 0-2) incidence occurred significantly more often in IS strategy participants $(107,55.2 \%)$ than in DS strategy participants (20, 39.2\%; $\mathrm{P}=0.04)$. Numbers and incidence of TIMI/ TMPG are shown in Table 2. Relative risk (RR) for the primary endpoint of TIMI was 0.55 [95\% confidence interval (CI): $0.20-1.44]$ and for TMPG it was 0.56 (95\% CI: 0.28-0.98).

\section{Secondary endpoints}

For secondary endpoints of MACEs, in-hospital MI occurred in 7 (3.6\%) IS strategy participants and in $1(2.0 \%)$ DS strategy participant $(\mathrm{RR}=0.53,95 \% \mathrm{CI}: 0.06-4.44$, $\mathrm{P}=0.56$ ). Furthermore, in-hospital cardiac death occurred in 3 IS strategy participants (1.5\%) and in no DS strategy participants; in-hospital all-cause mortality occurred in 3 IS participants (1.5\%) and in no DS group participants; inhospital heart failure occurred in $6(3.1 \%)$ compared with 0 
Table 1 Baseline clinical characteristics in participants with 2 strategies

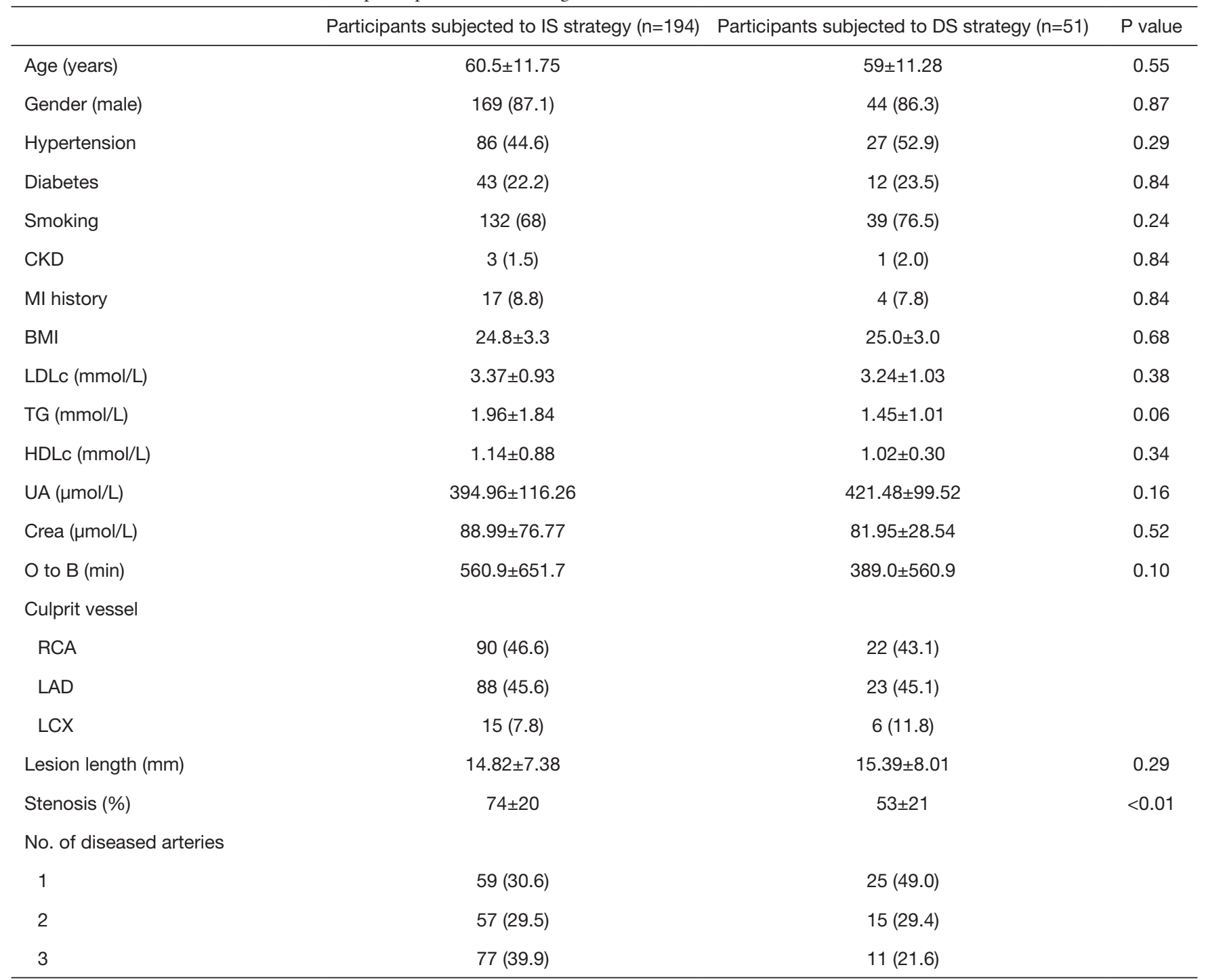

Data are shown as median (IQR) or n (\%). IS, immediate percutaneous stenting; DS, deferred stenting; CKD, chronic kidney disease; MI, myocardial infarction; BMI, body mass index; LDLc, low-density lipoprotein cholesterol; TG, triglycerides; HDLc, high-density lipoprotein cholesterol; UA, uric acid; Crea, creatinine; O to B, time from the symptom onset to balloon; Stenosis (\%), percentage of stenosis of ischemic target lesion. RCA, right coronary artery; LAD, left anterior descending artery; LCX, left circumflex artery.

Table 2 Primary outcomes of no-/slow-flow after stenting or final intervention

\begin{tabular}{|c|c|c|c|}
\hline & Participants in the IS strategy group & Participants in the DS strategy group & $P$ value \\
\hline No-/slow-reflow of TMPG & $107(55.2)$ & $20(39.2)$ & 0.04 \\
\hline
\end{tabular}

Data are shown as $\mathrm{n}(\%)$. IS, immediate stenting; DS, deferred stenting; TMPG, tissue myocardial perfusion grade. 
Table 3 Primary outcomes of TIMI/TMPG flow grade after stenting or final intervention

\begin{tabular}{|c|c|c|c|}
\hline & Participants in the IS strategy group & Participants in the DS strategy group & $P$ value \\
\hline 0 & $1(0.5)$ & $0(0)$ & \\
\hline 1 & $6(3.1)$ & $0(0)$ & \\
\hline 2 & $26(13.4)$ & $5(9.8)$ & \\
\hline TMPG after stenting or final intervention & & & $<0.01$ \\
\hline 0 & $8(4.1)$ & $0(0)$ & \\
\hline 1 & $83(42.8)$ & $0(0)$ & \\
\hline 2 & $17(8.8)$ & $20(39.2)$ & \\
\hline
\end{tabular}

Data are shown as n (\%). TIMI, thrombolysis in myocardial infarction; IS, immediate stenting; DS, deferred stenting; TMPG, tissue myocardial perfusion grade.

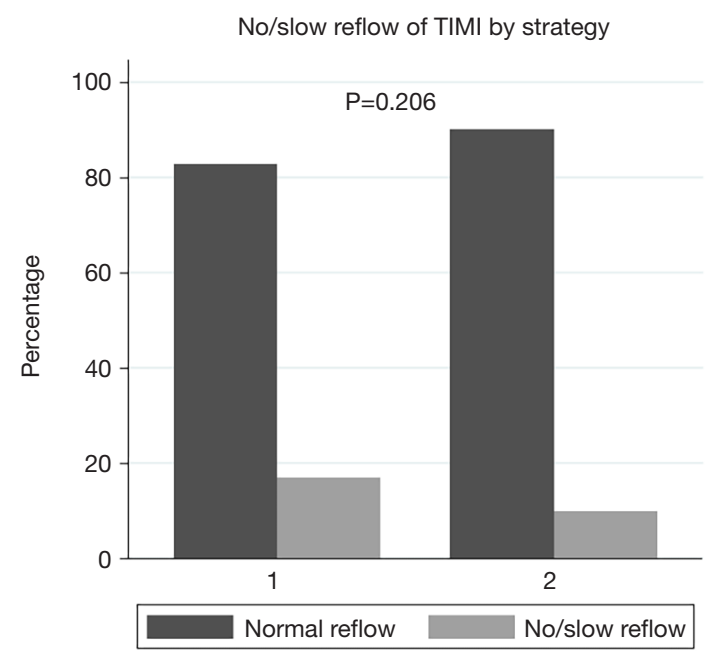

Figure 1 No statistical difference in occurrence of no-/slowreflow by TIMI flow grade between the two strategies. TIMI, thrombolysis in myocardial infarction.

(0\%) participants in the IS and DS groups, respectively; inhospital bleeding occurred in 4 participants from the IS group $(2.1 \%)$ and $2(3.9 \%)$ from the DS group, but the difference between groups was not significant $(\mathrm{P}=0.61)$ (Table 4).

Moreover, at 30-day follow-up, there was 1 IS strategy participant in whom an acute MI resulted from non-IRA. Heart failure (HF)-induced readmission occurred in 2 $(1.2 \%)$ and 1 participant (2.2\%) in the IS and DS groups,

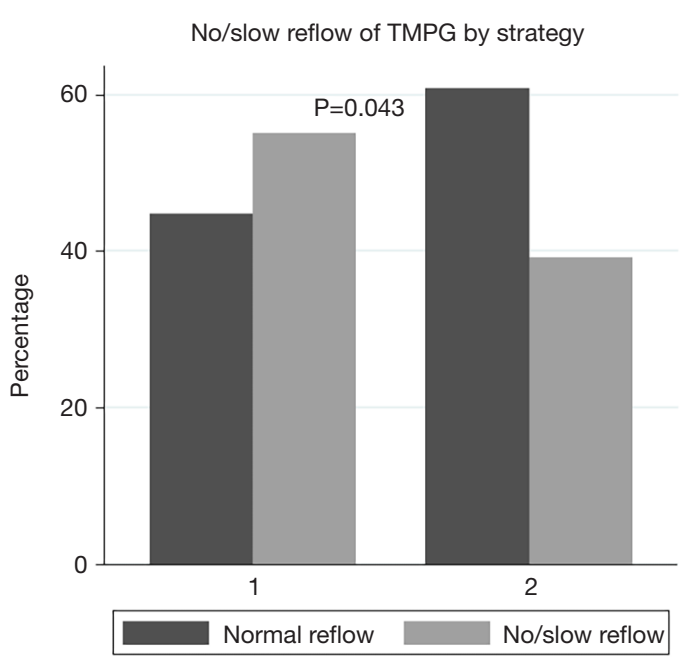

Figure 2 Reduced incidence of no-/slow-reflow by TMPG flow grade in DS strategy participants compared with IS strategy participants. TMPG, TIMI myocardial perfusion grade; DS, deferred stenting; IS, immediate stenting.

respectively $(\mathrm{P}=0.52)$. There were no events of cardiac death, all-cause-mortality, revascularization, or bleeding (Table 5). At 90-day follow-up, there were 4 participants $(2.7 \%)$ from the IS group in whom HF-induced readmission occurred. A single IS strategy participant $(0.7 \%)$ had a bleeding event (nasal hemorrhage) (Table 6).

\section{Other findings}

The majority of participants $(238,97.1 \%)$ underwent 
Table 4 Secondary outcomes of MACEs

\begin{tabular}{|c|c|c|c|}
\hline & Participants in the IS strategy group & Participants in the DS strategy group & $P$ value \\
\hline In-hospital CD & $3(1.5)$ & $0(0)$ & 0.37 \\
\hline In-hospital ACM & $3(1.5)$ & $0(0)$ & 0.37 \\
\hline In-hospital HF & $6(3.1)$ & $0(0)$ & 0.35 \\
\hline
\end{tabular}

Data are shown as n (\%). MACEs, major adverse cardiovascular events; IS, immediate stenting; DS, deferred stenting; MI, myocardial infarction; $\mathrm{CD}$, cardiac death; ACM, all-cause-mortality; HF, heart failure.

Table 5 Secondary outcomes of MACEs (followed-up at 30 days)

\begin{tabular}{|c|c|c|c|}
\hline & Participants in the IS strategy group & Participants in the DS strategy group & $P$ value \\
\hline CD (30 days) & $0(0)$ & $0(0)$ & - \\
\hline ACM (30 days) & $0(0)$ & $0(0)$ & - \\
\hline HF-induced rehospitalization (30 days) & $2(1.2)$ & $1(2.2)$ & 0.52 \\
\hline Revascularization (30 days) & $0(0)$ & $0(0)$ & - \\
\hline
\end{tabular}

Data are shown as $\mathrm{n}$ (\%). MACEs, major adverse cardiovascular events; IS, immediate stenting; DS, deferred stenting; MI, myocardial infarction; CD, cardiac death; ACM, all-cause-mortality; HF, heart failure.

Table 6 Secondary outcomes of MACEs (followed-up at 90 days)

\begin{tabular}{|c|c|c|c|}
\hline & Participants in the IS strategy group & Participants in the DS strategy group & $P$ value \\
\hline CD (90 days) & $0(0)$ & $0(0)$ & - \\
\hline ACM (90 days) & $0(0)$ & $0(0)$ & - \\
\hline HF-induced rehospitalization (90 days) & $4(2.7)$ & $0(0)$ & 0.58 \\
\hline Revascularization (90 days) & $0(0)$ & $0(0)$ & - \\
\hline
\end{tabular}

Data are shown as $\mathrm{n}$ (\%). MACEs, major adverse cardiovascular events; IS, immediate stenting; DS, deferred stenting; MI, myocardial infarction; $\mathrm{CD}$, cardiac death; ACM, all-cause-mortality; HF, heart failure.

echocardiography exams within $24 \mathrm{~h}$ after pPCI. The IS and DS strategy participants showed a comparable mean ejection fraction (EF) $(52.11 \%$ vs. $50.49 \%$, respectively; $\mathrm{P}=0.38)$, left ventricular diameter (48.35 vs. $48.77 \mathrm{~mm}$, respectively; $\mathrm{P}=0.65)$, and hospitalization costs $(\$ 9,727.4$ vs. $\$ 10,256$, respectively; $\mathrm{P}=0.74$ ) (Table 7).

\section{Discussion}

We showed that the DS strategy within $48-72 \mathrm{~h}$ after primary reperfusion did not improve TIMI flow grade $(17 \%$ vs. 9.8\%, $\mathrm{P}=0.21)$. This finding is in disagreement with previous studies in which DS strategy provided benefits for patients with high thrombus burden (5-8).

No-/slow-reflow phenomenon may result from several reasons including very high thrombus burden, embolizationrelated microvascular disturbance, endothelium dysfunction, or myocardial edema. Previous studies have shown that 
Table 7 Outcomes of echocardiography and hospitalization costs

\begin{tabular}{|c|c|c|c|}
\hline & Participants in the IS strategy group & Participants in the DS strategy group & $P$ value \\
\hline LVDD (mm) & $48 \pm 6$ & $49 \pm 5$ & 0.65 \\
\hline Hospitalization costs (\$) & $9,789 \pm 10,532$ & $10,321 \pm 7,846$ & 0.74 \\
\hline
\end{tabular}

Data are shown as median (IQR) or $\mathrm{n}(\%)$. IS, immediate stenting; DS, deferred stenting; LVEF, left ventricle ejection fraction; LVDD, left ventricle diastole diameter.

despite the patency of IRA and reperfusion to a TIMI flow grade 2-3 after PTCA with or without thrombus aspiration, the incidence of no-/slow-reflow might still increase in STEMI patients after IS compared with DS, which may confer worse prognosis. Instability of thrombus as well as erosion or rupture of vulnerable plaque are likely exacerbated when stents are placed immediately, because mechanical compression could possibly cause microvascular embolism by fragments mobilized from the aforementioned abnormality. Repeated aspiration thrombectomy or GP IIb/IIIa inhibitors through intra-coronary or intra-venous approach are possible alternatives in improving blood flow when facing to high thrombus burden or no-/slow- reflow according to previous studies and guidelines $(3,4,12,14,15)$. Sorts of vasodilators such as nitrates, nitroprusside or adenosine may be useful from experience. A feasible and effective solution may be a DS approach based on clinical status following a certain period of time with sufficient antithrombotic therapy when reperfusion is achieved. In the DEFER-STEMI study, worse blood flow of TIMI flow grade 0-2 was more commonly seen in patients treated with IS than in those treated by DS. Of note, that study included patients with high thrombus burden as well as other high-risk factors, including previous MI, older age, long lasting symptoms, and abnormalities of IRA (5-8). However, in the present study, we found no significant difference in TIMI flow grade between the 2 strategies, which contradicts the previous studies. Possible reasons for improved blood flow in epicardial arteries with high loading thrombus might not only include timely management of "D-to-B" and comprehensive pharmacotherapy such as antithrombotic therapy of loading dose dual-antiplatelettherapy, anticoagulants or platelet glycoprotein (GP) IIb/ IIIa inhibitors, but also wide use of interventional technical instruments or new-generation drug eluting stents $(12,16-18)$.

As far as we know, the meaning of "restoration of blood flow" is not only for the improved blood flow of epicardial coronary arteries upstream but also for the reperfusion of myocardial microvasculature downstream $(19,20)$.
Cardiac magnetic resonance (cMR), echocardiographic perfusion images, and TMPG are thought to be favorable choices to detect the myocardial perfusion (21). We particularly employed TMPG as a grading standard to represent microcirculation status. In our study, an obvious improvement of TMPG was discovered in DS strategy participants compared with the IS strategy $(55.2 \%$ vs. $39.2 \%, \mathrm{P}=0.04)$. Conflicting results exist among several previous studies $(7,9)$. However, based on the pathophysiological mechanism of IRA and our findings, we presume that DS strategy might play a protective role. We consider that in a strategy of DS, prolong duration (48-72 h) of effective dual-antiplatelet agents plus GP IIb/ IIIa inhibitors use do play an important part in reducing intra-arterial thrombus that distributed in epicardial vessels as well as microvasculature. Therefore, high thrombus loading condition could be relieved without compressing the vulnerable culprit lesion or coagula, avoiding worsen microcirculation from mobilizing fragments $(12,14-16)$.

We also did not find any significant reduction in incidence of MI, cardiac death, all-cause mortality, HFinduced readmission, repeated revascularization, or bleeding either during the in-hospital period or at 30- or 90-day follow-up in DS strategy participants compared with IS after STEMI of high thrombus burden. These results are comparable with previous findings from other studies $(10,11,19)$. No-/slow-reflow as well as microvasculature perfusion have been regarded as risk factors for poor prognosis $(3,5-8)$. In this study, we found no significant difference in MACEs between the 2 strategies, which might reflect the employment of improved medication with superior efficiency and standardized operations. We performed the optimal antithrombotic treatment including dual-antiplatelet therapy (aspirin plus clopidogrel/ticagrelor with IIb/IIIa receptor antagonist), which might lead to rapid dissolving of the residual thrombus after PCI with or without stent implantation. Though IS strategy participants showed significantly more events of no-/slow-reflow in 
TMPG, contemporary sufficient antithrombotic therapy may play an important role in alleviating microcirculatory obstruction within 24-72 h. During this time, swelling and edema of cardiomyocytes and endotheliocytes may be relieved simultaneously with removal of microvascular particles and debris. Intensified statin, $\beta$-blockers, as well as ACEI/ARBs were administered individually. However, no significant difference in ischemic events either during the n-hospital period or at follow-up of 30 or 90 days was found between participants treated with the 2 different strategies in our study. Furthermore, during the procedures performed on all participants, the use of interventional instruments consisting of guidewires, guiding catheters, aspirating catheters, and balloons was at the discretion of well-trained and equally skilled operators who were blinded to the trial. In this study, all participants with stent implantation received second-generation drug-eluting stents, which are thought to reduce intra-stent restenosis or acute in-stent thrombotic events when compared with firstgeneration drug-eluting stents in previous studies (22-24). Therefore, on the premise of employing sufficiently improved medical therapies and performing standardized operation with developed instruments, MACEs were comparable between participants with 2 different strategies.

In this study, cardiac function was evaluated by echocardiography. However, there was no significant difference between the 2 strategies in LVEF and left ventricular diastolic diameter (LVDD) $(\mathrm{P}=0.65)$. Such findings are in disagreement with other trials. Namely, in DANAMI-3 trial (10), LVEF was slightly higher in the DS group than in the IS group. Contrary to other studies, patients in our study were selected and characterized by high thrombus burden (TS $>2$ ). We anticipated that patients with STEMI with high thrombus burden might theoretically have lower LVEF or enlarged LVDD if TMPG no-/slow-reflow occurred after reperfusion through IS/DS strategy. However, there was no significant difference either in LVEF or LVDD despite worse microcirculation in patients with IS. Possible reasons may include timely reperfusion of IRA, optimized antiplatelet treatment, as well as early use of ACEI/ARB.

We performed the second procedure in participants with DS strategy and found that 21 of 51 patients (41.2\%) did not need stenting considering their mild to moderate residual stenosis and satisfying TIMI blood flow. There were 2 of $21(9.5 \%)$ cases that had in-hospital bleeding and 1 of $21(4.8 \%)$ cases that had HF-induced readmission but without other MACEs in participants without stenting during the in-hospital period and follow-up of 30 and 90 days. As far as we know, STEMI might result from rupture or erosion of vulnerable plaques or calcified nodules $(25,26)$. Numerous patients with STEMI could be diagnosed with lesions of mild-to-moderate stenosis before thrombus formation and perfusion loss. After intensive statin therapy and antiplatelet treatment, vulnerable plaques may be stabilized gradually, and subsequently the symptoms may be relieved. However, further studies are needed to determine whether a non-stent strategy could be a reasonable choice based on objective angiographic findings. In order to do so, intra-lumen technologies, intravenous ultrasound (IVUS), optical coherence tomography (OCT), for instance, which are gradually developing and being widely use nowadays, should be utilized abundantly to obtain accurate morphologic and structural evidence. Meanwhile, attention to predictors evaluation of high thrombus burden in pPCI such as older age, previous myocardial infarction or monocyte count should also be paid ahead so as to optimize the comprehensive management $(27,28)$.

\section{Study limitations}

First, since our study was conducted as an observational and non-randomized study, selection bias cannot be excluded. However, to recruit participants we chose universal inclusion criteria that did not differ from other trials, and both medical and interventional managements were homogenous. At the same time, baseline characteristics were comparable between participants of the 2 different strategies. Second, we did not perform a second angiography in IS participants, so dynamic evolution could not be evaluated after the index procedure. In order to exclude recurrent ischemia, we evaluated ECG and cardiac biomarkers during hospitalization in all participants to validate the stability of hemodynamics. Third, we used TMPG but not cardiac magnetic resonance imaging (cMRI) or myocardial perfusion ultrasound to assess microvascular obstruction precisely. For evaluating cardiac function, we assessed LVEF and LVDD, which are good indicators of cardiac function and are available in several hospitals instead of cMRI. Fourth, sample size of our study were small. According to incidence of no-/slow- reflow events in previous research, we calculated and set a goal in a total of 106 participants. At the end of trial, we finally recruited 245 patients. However, as a prospective clinical trial, we tend to plan and perform RCTs with larger number of patients in future, through which our conclusion would be validated 
perfectly. Fifth, we performed follow-ups at 30 or 90 days, which was short- to mid- term prognosis to evaluate the MACEs. Follow-up is still continuing, and new information of prognosis will be collected.

\section{Conclusions}

The DS strategy in STEMI patients with high thrombus burden reduced the risk of no-/slow-reflow in TMPG flow grade and might be beneficial for microcirculation, although it did not improve TIMI. The DS strategy did not reduce the risk of MI, cardiac death, all-cause mortality, revascularization, heart failure-induced readmission, bleeding events, or impaired cardiac function. A certain number of patients may be able to avoid stent implantation through employment of the DS strategy. However, more studies are needed to further evaluate the benefits of DS for microcirculation of certain subgroups of STEMI patients, such as in those with high thrombus burden. Moreover, the feasibility of selecting patients to avoid stenting must be further investigated.

\section{Acknowledgments}

This research was funded and supported by the National Key Research and Development Program of China, Grant (2016YFC1301202). We thank the staff of the Department of Cardiology at Guangdong Cardiovascular Institute. We extend thanks to LetPub (www.letpub.com) for its linguistic assistance during the preparation of this manuscript.

Funding: This study was funded by the National Key Research and Development Program of China, Grant (2016YFC1301202).

\section{Footnote}

Reporting Checklist: The authors have completed the STROBE reporting checklist. Available at http://dx.doi. org/10.21037/atm-21-1130

Data Sharing Statement: Available at http://dx.doi. org/10.21037/atm-21-1130

Conflicts of Interest: All authors have completed the ICMJE uniform disclosure form (available at http:// dx.doi.org/10.21037/atm-21-1130). All authors report that this study was funded by the National Key Research and Development Program of China, Grant
(2016YFC1301202). The authors have no other conflicts of interest to declare.

Ethical Statement: The authors are accountable for all aspects of the work in ensuring that questions related to the accuracy or integrity of any part of the work are appropriately investigated and resolved. This study was approved by the Ethics Committee of Guangdong Provincial People's Hospital with [GDREC2018346H(R2)]. All participants were informed about the purpose of the study and written informed consent was provided by all individual participants included in the study. All procedures performed in this study involving human participants were in accordance with the Declaration of Helsinki (as revised in 2013).

Open Access Statement: This is an Open Access article distributed in accordance with the Creative Commons Attribution-NonCommercial-NoDerivs 4.0 International License (CC BY-NC-ND 4.0), which permits the noncommercial replication and distribution of the article with the strict proviso that no changes or edits are made and the original work is properly cited (including links to both the formal publication through the relevant DOI and the license). See: https://creativecommons.org/licenses/by-nc-nd/4.0/.

\section{References}

1. Grines CL, Browne KF, Marco J, et al. A comparison of immediate angioplasty with thrombolytic therapy for acute myocardial infarction. The Primary Angioplasty in Myocardial Infarction Study Group. N Engl J Med 1993;328:673-9.

2. Stone GW, Grines CL, Browne KF, et al. Predictors of in-hospital and 6-month outcome after acute myocardial infarction in the reperfusion era: the Primary Angioplasty in Myocardial Infarction (PAMI) trail. J Am Coll Cardiol 1995;25:370-7.

3. Reffelmann T, Kloner RA. The "no-reflow" phenomenon: basic science and clinical correlates. Heart 2002;87:162-8.

4. Brener SJ, Dambrink JH, Maehara A, et al. Benefits of optimising coronary flow before stenting in primary percutaneous coronary intervention for ST-elevation myocardial infarction: insights from INFUSE-AMI. EuroIntervention 2014;9:1195-201.

5. Ndrepepa G, Tiroch K, Fusaro M, et al. 5-year prognostic value of no-reflow phenomenon after percutaneous coronary intervention in patients with acute myocardial 
infarction. J Am Coll Cardiol 2010;55:2383-9.

6. Tang L, Zhou SH, Hu XQ, et al. Effect of delayed vs immediate stent implantation on myocardial perfusion and cardiac function in patients with ST-segment elevation myocardial infarction undergoing primary percutaneous intervention with thrombus aspiration. Can J Cardiol 2011;27:541-7.

7. Carrick D, Oldroyd KG, McEntegart M, et al. A randomized trial of deferred stenting versus immediate stenting to prevent no- or slow-reflow in acute STsegment elevation myocardial infarction (DEFERSTEMI). J Am Coll Cardiol 2014;63:2088-98.

8. Ke D, Zhong W, Fan L, et al. Delayed versus immediate stenting for the treatment of ST-elevation acute myocardial infarction with a high thrombus burden. Coron Artery Dis 2012;23:497-506.

9. Lonborg J, Engstrom T, Ahtarovski KA, et al. Myocardial Damage in Patients With Deferred Stenting After STEMI: A DANAMI-3-DEFER Substudy. J Am Coll Cardiol 2017;69:2794-804.

10. Kelbaek H, Hofsten DE, Kober L, et al. Deferred versus conventional stent implantation in patients with STsegment elevation myocardial infarction (DANAMI 3-DEFER): an open-label, randomised controlled trial. Lancet 2016;387:2199-206.

11. Belle L, Motreff P, Mangin L, et al. Comparison of Immediate With Delayed Stenting Using the Minimalist Immediate Mechanical Intervention Approach in Acute ST-Segment-Elevation Myocardial Infarction: The MIMI Study. Circ Cardiovasc Interv 2016;9:e003388.

12. Savonitto S, De Luca G, Goldstein P, et al. Antithrombotic therapy before, during and after emergency angioplasty for ST elevation myocardial infarction. Eur Heart J Acute Cardiovasc Care 2017;6:173-90.

13. Mehran R, Rao SV, Bhatt DL, et al. Standardized bleeding definitions for cardiovascular clinical trials: a consensus report from the Bleeding Academic Research Consortium. Circulation 2011;123:2736-47.

14. Burzotta F, Trani C, Romagnoli E, et al. A pilot study with a new, rapid-exchange, thrombus-aspirating device in patients with thrombus-containing lesions: the Diver C.E. study. Catheter Cardiovasc Interv 2006;67:887-93.

15. Ibanez B, James S, Agewall S, et al. 2017 ESC Guidelines for the management of acute myocardial infarction in patients presenting with ST-segment elevation. Kardiol Pol 2018;76:229-313.

16. Parker WA, Storey RF. Long-term antiplatelet therapy following myocardial infarction: implications of
PEGASUS-TIMI 54. Heart 2016;102:783-9.

17. Garg A, Brodie BR, Stuckey TD, et al. New generation drug-eluting stents for ST-elevation myocardial infarction: a new paradigm for safety. Catheter Cardiovasc Interv 2014;84:955-62.

18. Alawami M, Sadler M, Kasargod C, et al. Outcomes of patients with ST elevation myocardial infarction in the era of second-generation drug eluting stents; five-year followup. N Z Med J 2019;132:34-41.

19. Ito H, Tomooka T, Sakai N, et al. Lack of myocardial perfusion immediately after successful thrombolysis. A predictor of poor recovery of left ventricular function in anterior myocardial infarction. Circulation 1992;85:1699-705.

20. Ito H, Maruyama A, Iwakura K, et al. Clinical implications of the 'no reflow' phenomenon. A predictor of complications and left ventricular remodeling in reperfused anterior wall myocardial infarction. Circulation 1996;93:223-8.

21. Bouleti C, Mewton N, Germain S. The no-reflow phenomenon: State of the art. Arch Cardiovasc Dis 2015;108:661-74.

22. Kedhi E, Gomes ME, Lagerqvist B, et al. Clinical impact of second-generation everolimus-eluting stent compared with first-generation drug-eluting stents in diabetes mellitus patients: insights from a nationwide coronary intervention register. JACC Cardiovasc Interv 2012;5:1141-9.

23. Tada T, Byrne RA, Simunovic I, et al. Risk of stent thrombosis among bare-metal stents, first-generation drug-eluting stents, and second-generation drug-eluting stents: results from a registry of 18,334 patients. JACC Cardiovasc Interv 2013;6:1267-74.

24. Philip F, Agarwal S, Bunte MC, et al. Stent thrombosis with second-generation drug-eluting stents compared with bare-metal stents: network meta-analysis of primary percutaneous coronary intervention trials in STsegment-elevation myocardial infarction [corrected]. Circ Cardiovasc Interv 2014;7:49-61.

25. Chang J, Nair V, Luk A, et al. Pathology of myocardial infarction. Diagnostic Histopathology 2013;19:7-12.

26. Pasotti M, Prati F, Arbustini E. The pathology of myocardial infarction in the pre- and post-interventional era. Heart 2006;92:1552-6.

27. Wang Z, Liu N, Ren L, et al. Association of Monocyte Count on Admission with the Angiographic Thrombus Burden in Patients with ST-Segment Elevation Myocardial Infarction Undergoing Primary Percutaneous Coronary 
Page 12 of 12

Intervention. Arq Bras Cardiol 2018;110:333-8.

28. Arisoy A, Altunkas F, Karaman K, et al. Association of the Monocyte to HDL Cholesterol Ratio With Thrombus Burden in Patients With ST-Segment Elevation Myocardial Infarction. Clin Appl Thromb Hemost

Cite this article as: Luo $\mathrm{D}, \mathrm{Hu} \mathrm{X}$, Sun S, Wang C, Yang $\mathrm{X}$, Ye J, Guo X, Xu S, Sun B, Dong H, Zhou Y. The outcomes in STEMI patients with high thrombus burden treated by deferred versus immediate stent implantation in primary percutaneous coronary intervention: a prospective cohort study. Ann Transl Med 2021;9(7):573. doi: 10.21037/atm-21-1130
Luo et al. DS or IS in STEMI with high thrombus burden

2017;23:992-7.

(English Language Editor: J. Jones) 